\title{
Las aptitudes vocacionales de las
} mujeres

\section{Leta Stetter Hollingworth}

\section{Traducción: Adriana Velásquez y María Ponce de León}

(Publicación original: Hollingworth, L. S. (1916). The vocational aptitudes of women. En Harry Hollingworth (ed.). Vocational Psychology (pp. 222-245) New York: D. Appleton).

Es usual que los autores, cuando discuten sobre problemas vocacionales, asuman que el futuro vocacional de las jóvenes está determinado a priori por el sexo. En los textos se señala con frecuencia la falta de recursos para la formación doméstica en nuestros institutos y universidades y se hacen sugerencias sobre cómo establecer las artes y ciencias domésticas, con una base más sólida, dentro del sistema educativo. Algunos de los párrafos pueden estar dedicados a la discusión de las estadísticas que muestran que miles de muchachas van de la escuela a la industria, y a la pregunta sobre qué tipo de formación es más adecuada para ayudarlas a ganarse la vida en el período que transcurre entre la graduación y el matrimonio. Así termina la discusión sobre los problemas vocacionales, en lo que respecta a las muchachas, y el resto se dedica a consideraciones más pertinentes sobre las aptitudes vocacionales y la orientación de los muchachos.

Este capítulo tiene como objetivo preguntarnos si existen diferencias esenciales e innatas entre los sexos, en lo que respecta a los gustos y habilidades, que pudieran ofrecer una base científica para la suposición tradicional y, aparentemente arbitraria, de que el futuro vocacional de las muchachas debe enmarcarse por naturaleza dentro del ámbito doméstico, y por ello no presenta problemas; mientras que el futuro de los muchachos es completamente problemático y puede perfilarse en una veintena de profesiones diferentes dependiendo de las aptitudes personales. Intentaremos determinar si la expectativa actual de que todas las mujeres seguirán la vocación del cuidado del hogar está fundamentada en algún hecho o hechos del intelecto humano, o si simplemente surge a partir de ideas de la conveniencia tradicional vinculadas al cuidado de los pequeños $y$, también, si esto conduce a un desperdicio de energía y talentos intelectuales.

La discusión consistirá en cinco preguntas generales junto a sus respuestas a la luz de la psicología experimental: (1) ¿Existen diferencias innatas entre los sexos respecto a la inteligencia media? (2) ¿Alguno de los sexos presenta más variedad en cuanto a los rasgos mentales? (3) ¿La incapacidad intelectual obedece a una causa particular que afecta a un sexo y no al otro? (4) ¿Existen diferencias entre los sexos respecto al bagaje afectivo o instintivo que pudieran conducir naturalmente a una diferenciación vocacional entre los sexos? (5) ¿Qué explicación se le puede dar a la división tradicional del trabajo entre los sexos?

Será necesario, de entrada, establecer una clara distinción entre la bibliografía de opinión y la bibliografía de hechos. La bibliografía de opinión incluye todas aquellas afirmaciones, hechas por hombres de ciencia y otros, que no están basadas en la evidencia experimental. La bibliografía de 
opinión sobre el tema de las diferencias entre los sexos respecto a los rasgos mentales es cuantiosa. Aparece en los escritos de Nietzsche, Schopenhauer, Mill, Möbius y otros. Por bibliografía de hechos se entiende todas aquellas afirmaciones que están basadas en datos experimentales, los cuales han sido obtenidos bajo condiciones cuidadosamente controladas y que pueden ser verificados por cualquier persona competente para comprenderlos y evaluarlos. En este capítulo buscaremos las respuestas a las preguntas propuestas sólo en la bibliografía de hechos e ignoraremos toda la bibliografía de opinión por considerarla irrelevante para la discusión.

Puesto que la discusión se limitará a la bibliografía de hechos, será necesario referirse sólo a la bibliografía relativamente reciente. Hasta hace unos quince años no había habido prácticamente ningún intento de recopilar datos precisos sobre el tema de las diferencias entre los sexos respecto a sus capacidades mentales. Antes de que se intentara conseguir los datos experimentales, se aceptaba la hipótesis de que las mujeres eran intelectualmente, por naturaleza, diferentes e inferiores a los hombres. El factor del sexo era determinante; la manera de descubrir si un individuo dado era capaz de realizar una tarea intelectual específica no consistía en dejar que el individuo realizara la tarea y luego juzgar por los resultados, sino en indicar el sexo de la persona en cuestión.

Paralelamente a la intensa controversia que se suscitó en el siglo xix sobre la educación superior de las mujeres, se llevaron a cabo una serie de estudios estadísticos utilizando el método del cuestionario. A éstos les siguieron los estudios experimentales $\mathrm{y}$, a comienzos del siglo $\mathrm{xx}$, se realizaron varios experimentos para investigar las diferencias intelectuales entre los sexos. En ese momento también comenzó a prosperar la idea de que cualesquiera que fueran las diferencias entre los sexos, tal como las encontramos en el mundo, pudieran deberse a la educación y no a la naturaleza original; y comenzó a destacarse que este aspecto del problema complica incluso a la investigación experimental en aspectos difíciles de controlar.

En este punto nos podemos referir a los experimentos que medían el peso del cerebro, publicados y ampliamente discutidos hace unos treinta años. Romanes, entre otros, insistía que el cerebro masculino era, de media, algunos gramos más pesado que el cerebro femenino, y por un tiempo se supuso que el hecho de la inferioridad innata de las mujeres quedaba, de esta manera, establecido satisfactoriamente. Sin embargo, posteriormente se demostró que, en relación con el peso total del cuerpo, el cerebro femenino es igual de pesado que el masculino. También se descubrió que no se podía establecer una correlación positiva entre el peso del cerebro y el intelecto.

En 1906, Helen Bradford Thompson publicó, en la Universidad de Chicago, su tesis doctoral titulada The Mental Traits of Sex. Este texto contiene un compendio de los pocos trabajos experimentales realizados antes de esa fecha y presenta un gran número de experimentos realizados a un grupo de hombres y mujeres en la Universidad de Chicago. El resultado de las pruebas realizadas sobre varios rasgos mentales es que las diferencias entre los sexos en ningún caso eran mayores a las diferencias individuales dentro del mismo sexo. En estos experimentos, los hombres se diferenciaban entre ellos (así como las mujeres entre ellas), tanto como los hombres se diferenciaban de las mujeres. Sólo en dos de los rasgos sometidos a prueba se encontraron diferencias sustanciales entre las tendencias generales de los sexos. En la velocidad del movimiento voluntario, los hombres eran más rápidos que las mujeres, y en la memoria, las mujeres eran superiores a los hombres. Tomados en su conjunto, 
los resultados indicaron que la habilidad mental era igual entre los sexos ${ }^{1}$. A los efectos de esta investigación será suficiente decir que después de veinte años recopilando datos para los experimentos científicos, la hipótesis de que existe alguna diferencia innata entre los sexos, en lo que respecta a la habilidad intelectual media, ha sido abandonada por todos los psicólogos que basan sus afirmaciones en la evidencia científica. Por ejemplo, el doctor E. L. Thorndike, en la última edición de Educational Psychology (1914), resume el trabajo experimental sobre las diferencias entre los sexos respecto a la inteligencia media:

"La característica más importante de estas diferencias es que son muy escasas. Las diferencias individuales dentro de cada sexo son mucho mayores que cualquier diferencia entre los sexos. Por ello, a efectos prácticos, tales diferencias pueden descartarse. [...] Como es bien sabido, los experimentos de la última generación concernientes a la educación de las mujeres han demostrado que éstas tienen la misma capacidad para realizar las actividades escolares en todos los niveles educativos: primario, secundario y universitario. [...] Las mediciones de los psicólogos nos llevan a la conclusión de que esta igualdad de rendimiento se debe a una igualdad de dones naturales y no a un esfuerzo compensatorio de los talentos menores de las mujeres."

De este modo, nuestra primera pregunta: ¿Existen diferencias innatas entre los sexos, en cuanto a la inteligencia media, que supondrían una diferenciación de las vocaciones basada en el sexo?, puede ser contestada de la siguiente manera. La bibliografía de hechos no ofrece datos sobre ninguna diferencia considerable entre los sexos en cuanto a la habilidad mental media. La evidencia de la ciencia experimental (y sobre este tema ya existe una gran cantidad de evidencia disponible) demuestra, a través de las pruebas de medias, que ambos sexos poseen la misma capacidad para realizar tareas mentales.

Nuestra segunda pregunta: ¿Existe alguna diferencia entre los sexos con respecto a la variabilidad de los rasgos mentales que pudiera suponer una diferenciación de las vocaciones basada en el sexo?, no ha sido investigada por la ciencia experimental tan exhaustivamente como la primera pregunta. Lo que intentamos descubrir es si al estudiar un rasgo mental determinado en dos grupos (iguales en número y seleccionados de la misma manera), uno de muchachos y otro de muchachas, los muchachos se diferenciarían unos de otros más de lo que las muchachas se diferenciarían entre sí. En otras palabras, ¿los miembros de un sexo son muy parecidos en sus gustos, intereses y habilidades, mientras que los miembros del otro sexo difieren ampliamente en cuanto a sus gustos, intereses y habilidades? Obviamente, este podría ser el caso, aunque los dos grupos consiguieran exactamente la misma puntuación media en tales rasgos. La respuesta a esta segunda pregunta sería de gran importancia para la orientación vocacional. Por ejemplo, si los datos experimentales demostraran que las mujeres son más bien parecidas entre sí, por naturaleza, mientras que los hombres se diferencian en un amplio rango unos de otros, tendríamos una base científica para concluir que la justicia y la economía social son correctas conforme a la política actual de orientar a

\footnotetext{
1 En el número correspondiente a octubre (1914) del Psychological Bulletin, se publicó un compendio de todo el trabajo experimental relevante que se había realizado en los últimos años sobre las diferencias entre los sexos. Cualquier lector que esté interesado en revisar con detalle los datos hará bien en consultar todas las referencias ofrecidas en el texto.
} 
todas las muchachas hacia una única ocupación, mientras que a los muchachos se les incentiva a acceder a la mayor variedad posible de profesiones.

El primero que opinó sobre la variabilidad comparativa de los sexos fue un anatomista llamado Meckel, hace casi un siglo. Es muy interesante (así como gracioso), a la luz de las ideas posteriores sobre la variabilidad, citar sus comentarios. Meckel pensaba que las mujeres eran más variables que los hombres y opinaba que: "puesto que la mujer es un animal inferior y la variabilidad es un signo de inferioridad", ¡la conclusión estaba justificada! Cincuenta años más tarde, cuando Darwin le dio un nuevo rostro a la variabilidad y demostró que era una ventaja y una característica que ofrecía la mayor esperanza de progreso, se comenzó a afirmar la mayor variabilidad del hombre en toda la bibliografía de opinión. Karl Pearson fue el único que se opuso a ese punto de vista, que era común en el siglo xix y aún es compartido por muchos, y afirmó que no existía hasta el momento ninguna bibliografía de hechos que mencionase la variabilidad comparativa (aunque los hombres de ciencia no se hubieran reprimido, por este motivo, de pronunciar los más favorables argumentos sobre este tema). Acto seguido, Pearson recogió y computó cientos de medidas de seres humanos, y presentó los resultados en 1897, en un exhaustivo artículo titulado: "Variation in Man and Woman". Pearson demostró con claridad que no existe ningún indicio de una mayor variabilidad masculina, cuando las medidas anatómicas de los seres humanos adultos son tratadas desde un punto de vista matemático. Inmediatamente después, Havelock Ellis, cuyas opiniones se vieron claramente afectadas por el artículo de Pearson, respondió que cuando los adultos se convierten en el sujeto de la investigación, no se puede obtener ninguna información sobre el origen de las diferencias de variabilidad. Ya que debido a las costumbres sociales, etc., el nacimiento, la vida y la muerte afectan a los sexos de manera desigual, nadie puede decir, en el caso de los adultos, cuánto influye el ambiente y cuánto se debe a la naturaleza original. Si Ellis hubiera tenido en cuenta esta crítica antes de escribir su libro, Man and Woman, el capítulo "The Variational Tendency of Men" ciertamente no habría sido publicado. Sin embargo, su crítica sobre el artículo de Pearson no es menos válida sólo porque no haya podido aplicarla a su propio trabajo. Es cierto que las medidas de los adultos no nos dicen nada sobre los bebés, los cuales aún no han sido sometidos a las influencias formativas y selectivas del ambiente y la educación. No obstante, el artículo de Pearson continuó siendo prácticamente la única bibliografía de hechos sobre la variabilidad anatómica comparativa entre los sexos hasta el año 1914. En ese año, se publicó un artículo de Montague y Hollingworth en el American Journal of Sociology donde se exponían las medidas de dos mil recién nacidos, un millar de cada sexo. El resultado de estas estadísticas demostró que no existe ninguna diferencia respecto a la variabilidad entre los sexos.

Podría parecer irrelevante hacer tanto hincapié en los datos anatómicos, cuando el propósito de este capítulo es tratar las aptitudes mentales. Sin embargo, la pertinencia de estos datos tiene que ver con el hecho de que si se puede establecer alguna diferencia en cuanto a la variabilidad física entre los sexos, esto sugeriría (aunque no probaría) también la existencia de una variabilidad mental entre los sexos. Nunca se han realizado estudios experimentales con el propósito específico de determinar si existen diferencias entre los sexos en términos de variabilidad mental. Los pocos datos que poseemos han llegado incidentalmente a través de otros estudios realizados con otro tipo de objetivos. Esos datos fueron recolectados y sintetizados en el American Journal of Sociology, en enero de 1914. En aquel momento había muy poca evidencia que pudiera ser citada sobre el tema y la que había no sentaba ninguna base para seguir sosteniendo la existencia de alguna diferencia respecto a la variabilidad mental entre los sexos. Desde el año 1914 han sido publicados los experimentos de Trabue, realizados a través de tests para completar frases, a unos 1300 niños en 
edad escolar; los tests de aritmética de Courtis, realizados en Nueva York a miles de niños en edad escolar; los estudios de Terman, a través de los tests de Binet-Simon, realizados a mil niños escogidos de manera aleatoria; $y$, los de Pyle, quien ha llevado a cabo su estudio sobre la medición de niños en edad escolar. La evidencia de estos exhaustivos experimentos confirma, en todos los casos y según las mediciones, que no existen diferencias respecto a la variabilidad mental entre los sexos.

Es importante que el lector tenga en mente que aún existe mucha controversia, tanto entre los mejores expertos preparados para comprender los problemas de variación, como en los propios métodos para medir la variabilidad comparativa. No es necesario explicar aquí las consideraciones matemáticas involucradas. Pero hasta que no haya sido determinado definitivamente cómo se puede medir científicamente la variabilidad comparativa, sería prematuro expresar alguna conclusión sobre las diferencias entre los sexos respecto a la variabilidad mental.

Por lo tanto, la respuesta a nuestra segunda pregunta sería la siguiente: no existe un acuerdo generalizado entre las personas más aptas para opinar sobre cuál es el método científico para medir la variabilidad relativa. Sin embargo, en lo que respecta a los numerosos rasgos estudiados, según los métodos que ahora se consideran los más fiables y según los estudios en los cuales presumiblemente se han utilizado los métodos más adecuados de medición, no existen motivos para suponer que hay diferencias entre los sexos en términos de variabilidad. Nunca se ha realizado un estudio experimental que recogiera muestras de ambos sexos de manera amplia, aleatoria, proporcionada y de grupos igualmente homogéneos tanto racial como socialmente, que mostrara alguna diferencia fiable entre los sexos en términos de variabilidad. Si nos adherimos a la bibliografía de hechos debemos concluir, por lo que sabemos hasta ahora, que las mujeres difieren entre ellas tanto como difieren los hombres entre sí en cuanto a habilidades y aptitudes.

Llegamos así a preguntarnos si la incapacidad intelectual obedece a una causa particular que afecta a un sexo y no al otro. Con relación a este asunto, podríamos tomar en consideración el período menstrual, que caracteriza a las mujeres jóvenes y adultas pero no caracteriza a los hombres jóvenes y adultos. La menstruación ha sido siempre objeto de supersticiones y tabúes, e incluso lo sigue siendo entre los pueblos más civilizados de la actualidad; la bibliografía de opinión está repleta de referencias a ella como fuente de debilidad intelectual e irresponsabilidad. Quizás deberíamos dejar hablar sobre este aspecto a Frederick Harrison en nombre de muchos otros escritores:

"Suponiendo que todas las demás fuerzas fueran idénticas, sólo el porcentaje de ineptitud producido por el período menstrual es el que marca la diferencia de la capacidad de trabajo entre los dos sexos. Este punto fundamental ha sido obviado debido a una aprensión natural a enfrentarse a los datos concretos y a un formalismo un tanto inapropiado".

La bibliografía de opinión abunda en diferentes nociones, incoherencias y ejemplos contradictorios en lo que se refiere a la menstruación y su supuesta gran influencia en la vida intelectual y profesional de las mujeres. Gran parte de la oposición a la educación de las mujeres se ha basado en este aspecto e incluso ha sido aprovechado como un buen motivo para negar la libertad política a las mujeres. Se ha expuesto fehacientemente que, por esta razón, las mujeres no son aptas para dedicarse a la vida profesional o comercial; sin embargo, no se propone que las cocineras, limpiadoras, madres, niñeras, amas de casa o bailarinas sean eximidas periódicamente de sus labores y responsabilidades. 
No se encuentra casi ninguna bibliografía de hechos donde se relacione el período menstrual con la habilidad mental de las mujeres. Hasta hace muy poco, no se había realizado esfuerzo alguno por estudiar este asunto utilizando instrumentos y métodos de precisión. Los psicólogos, que a menudo exponen que la influencia de la periodicidad en la mente es fundamental y característica, omitieron considerarla cuando realizaron estudios a mujeres. En 1909, Voitsecovsky realizó en Petrogrado un experimento con seis mujeres utilizando instrumentos de precisión. Creyó haber obtenido un resultado concluyente y evidencias que confirmaban la influencia real de la periodicidad en ciertas funciones mentales. Sus conclusiones, no obstante, carecen de validez en gran medida por el hecho de que todos los sujetos conocían el propósito del experimento y, además, porque no utilizó, a modo de control, seres humanos que no estuviesen sujetos al fenómeno en cuestión. También omitió presentar todos los datos y así la fiabilidad de sus conclusiones podía ser admitida.

En 1914, se presentaron dos estudios sobre este fenómeno. El primero era del Dr. A. E. Arnold con relación a los efectos del trabajo escolar sobre el período menstrual, el cual fue publicado en el número de enero del American Physical Education Review. Este investigador sospechaba, debido a su experiencia como médico y maestro, "que gran parte de la incapacidad aducida era ficticia", y decidió establecer, a modo de experimento, un régimen según el cual ningún alumno bajo su supervisión podría ser eximido periódicamente de sus obligaciones físicas o mentales, salvo los casos en donde existiera alguna condición patológica. Al recopilar los datos, afirmó: "Hasta el momento, todos nuestros resultados muestran mejorías (en la salud de los estudiantes)".

El segundo estudio, que apareció en 1914, fue realizado por quien esto escribe. La autora hizo un estudio experimental cuidadoso y prolongado sobre veintitrés mujeres (utilizando a modo de control los resultados obtenidos de los sujetos hombres), y no logró demostrar ningún tipo de influencia del período menstrual sobre las habilidades mentales que había analizado. Estas incluían: la velocidad y exactitud en la percepción, la asociación controlada, la atención, la velocidad de los movimientos voluntarios, la fatiga y la velocidad de aprendizaje.

Aún falta realizar una gran cantidad de investigaciones científicas antes de poder responder de algún modo la pregunta: ¿Ejerce el período menstrual una influencia fundamental y característica en las habilidades intelectuales de las mujeres? Debemos responder a la tercera pregunta de esta manera: existe muy poca evidencia experimental sobre la cual basar una posible respuesta, pero los pocos datos que sí tenemos no demuestran ningún tipo de influencia, ya sea ésta perjudicial o beneficiosa.

Nuestra cuarta pregunta es la siguiente: ¿Existe alguna diferencia innata entre los sexos, relacionada con el bagaje afectivo o instintivo, que condujera naturalmente a una diferenciación vocacional entre los sexos? En este punto, debemos admitir que no contamos con ningún tipo de bibliografía de hechos. La bibliografía de opinión es muy extensa sobre este tema y sería una tarea interesante y, sin duda, instructiva reunir y resumir las diversas y contradictorias opiniones de los hombres en lo que respecta a las diferencias afectivas e instintivas entre los dos sexos. Los hombres y mujeres, tal como los vemos en el mundo, tienen realmente un comportamiento emocional diferente, pero nadie puede decir si estas diferencias de comportamiento son originales o adquiridas. Existen diferentes normas convencionales de comportamiento emocional para hombres y mujeres, pero nadie tendría motivos justificados para decir que esas normas han surgido de las diferencias emocionales inherentes a los dos sexos. La misma diversidad que caracteriza las opiniones acerca de este tema constituye la prueba de la ignorancia de la humanidad en este campo. 
Debido a la falta total de datos, el debate sobre esta última pregunta no debiera entretenernos. Sin embargo, deberíamos observar un instinto, del cual se ha afirmado reiteradamente que caracteriza a la mujer y que constituye por sí mismo una justificación natural para diferenciar las vocaciones de los sexos: el "instinto maternal". Puesto que el período de indefensión de la primera infancia del ser humano es muy prolongado y el cuidado de los niños es una labor ardua y exigente, sería de esperar que aquellos individuos que no están biológicamente ligados a los niños utilizaran todos los medios a su alcance para asignar toda la responsabilidad de su cuidado a los que sí están originariamente ligados a éstos. Deberíamos esperar que esto sucediera, como de hecho sucede. Constantemente se ha llevado a cabo una campaña social para establecer como norma a la mujer cuyas inclinaciones vocacionales están completa y "naturalmente" satisfechas con la maternidad y la crianza de los niños.

A falta de datos, lo más razonable sería suponer que, si fuera posible obtener una medida cuantitativa del "instinto maternal", encontraríamos que este rasgo se encuentra distribuido entre las mujeres del mismo modo que hemos encontrado distribuidos todos los demás rasgos que sí han podido ser medidos cuantitativamente. Es totalmente razonable suponer que obtendríamos una curva de distribución que variaría desde un extremo, donde los individuos tienen un interés cero o negativo por el cuidado de los niños; atravesaría una media, donde existe un impulso moderado hacia el cuidado de los niños; hasta el otro extremo, donde el único interés vocacional yace en tal actividad. El alumbramiento y crianza de los niños se parece, en muchos aspectos, al trabajo de los soldados: es necesario para la existencia de la nación, implica grandes sacrificios personales, conlleva sufrimiento, peligro y, en cierto porcentaje de casos, incluso la muerte. Por lo tanto, como en el caso de los soldados, se deben realizar todos los esfuerzos necesarios para establecer como norma el extremo de la curva de distribución donde se encuentra un ferviente interés por el patriotismo, en este caso, y por la maternidad en el otro. A falta de datos científicos debemos evitar, por consiguiente, aceptar como hecho establecido de la naturaleza humana una doctrina que posiblemente encontraremos funcionando como medio de control social. También es adecuado preguntar qué significa exactamente el término "instinto maternal". ¿Se refiere al deseo de descendencia que aún no existe? ¿Se refiere sólo a la tendencia a proteger a la indefensa prole una vez que ésta ya existe? ¿Significa el interés por los niños como tales, sin tener en cuenta sus orígenes? ¿O consiste en la fusión de todos estos elementos? Sobre todo, ¿supone como elemento esencial, el interés por atender personalmente a los niños? Ciertamente, examinando la extensa biografía de opinión, se tiene la impresión de que para la mayoría de las personas el término es bastante confuso y requiere un análisis.

Ya hemos considerado cuatro de nuestras preguntas a la luz de la evidencia experimental. Hemos descubierto que todavía queda mucho trabajo por hacer antes de que podamos contestar de manera concluyente la mayoría de ellas y que al menos una de las preguntas no puede ser contestada por la bibliografía de hechos. Solamente podemos decir que, hasta ahora, los experimentos científicos no han revelado ninguna diferencia entre los sexos con respecto a la naturaleza del intelecto que implicaría una inevitable diferenciación vocacional basada en el sexo. No se encuentran datos científicos para demostrar: (1) diferencias en cuanto al intelecto medio; (2) diferencias respecto a la variabilidad mental; (3) causas especiales de la incapacidad intelectual que afecten a un sexo y no al otro; (4) diferencias con relación al bagaje afectivo o instintivo que impliquen una división "natural" del trabajo.

Por lo que sabemos, la división del trabajo entre los sexos, que ha existido desde tiempos inmemoriales y aún persiste, tiene su origen en las diferencias fisiológicas y no en las psicológicas. 
Nuestra organización económica y profesional se ha basado en la principal diferencia que existe entre los sexos: el hecho fisiológico trascendental de que son las mujeres las que dan a luz y alimentan a los niños y no los hombres. Podría suponerse que la selección natural ha desarrollado en las mujeres un rasgo intelectual (o no intelectual) que encontrará su completa satisfacción natural a través de la vocación de dar a luz y criar a los niños. Sin embargo, tal selección podría suceder sólo si los rasgos mentales se heredaran de acuerdo al sexo o si existieran como características secundarias de los sexos. No existen pruebas que demuestren que los rasgos mentales se heredan de acuerdo al sexo 0 que existen como caracteres sexuales secundarios. De acuerdo a lo que sabemos, las hijas heredan los rasgos mentales tanto de los padres como de las madres y los hijos los heredan de las madres tanto como de los padres. Bajo estas circunstancias, la ley de selección natural no podrá nunca ser válida para solucionar los problemas vocacionales de las mujeres.

Frecuentemente, se aduce como prueba de la incapacidad innata para llevar a cabo otras actividades que no sean las domésticas el hecho de que, en el pasado, las mujeres no han igualado a los hombres en "filosofía, ciencia, arte, inventiva y liderazgo". De tal prueba, sin embargo, no recogemos en realidad ningún tipo de información acerca de las aptitudes vocacionales de las mujeres. No se puede esperar de las mujeres ningún logro considerable en los campos anteriormente mencionados debido a los siguientes motivos. Las mujeres deben dar a luz y alimentar a los niños, algo que los hombres no pueden hacer. Además, el período de gestación y la etapa infantil son muy prolongados en la especie humana; para cada niño se emplean, en total, alrededor de seis años. Hasta hace poco tiempo no se conocía o utilizaba de manera general ningún método científico para controlar la procreación. Así, las mujeres han dado a luz a un gran número de hijos y han empleado toda su juventud y madurez para tenerlos y criarlos. Es muy poco probable que la pequeña minoría de mujeres cuyas vidas, por casualidad, no fueron consumidas de tal manera, hiciera alguna aportación fuera del campo doméstico. Esto se debe a dos razones. En primer lugar, se suponía que todas las mujeres debían formar pareja y así procrear y criar a los hijos; por lo tanto, la sociedad no proveía la formación en otros campos que no fueran en los que se suponía que se desarrollarían. En segundo lugar, aquellas mujeres que no tuvieron ese destino común, no lo hicieron por algún motivo especial: ya fuera porque tenían una mala salud, alguna enfermedad mental o la necesidad de cuidar a algún familiar decrépito. Las causas mismas de su celibato jugarían también en contra de cualquier logro profesional que alcanzaran por su parte.

Debido al método irracional de ensayo y error mediante el cual se han desarrollado nuestras instituciones humanas, sería lógico esperar que la gran diferencia fisiológica que existe entre los sexos con relación a su función reproductiva pudiera influenciar las actividades profesionales, tal y como lo ha hecho. En la división tradicional del trabajo entre los sexos encontramos exactamente lo que esperaríamos encontrar, incluso si las habilidades intelectuales e intereses fueran idénticos. Parece conveniente, tanto psicológica como socialmente, poner de relieve el único factor condicionante e indiscutible para la diferenciación vocacional entre hombres y mujeres, en lugar de sumergirlo, como se ha hecho anteriormente, por medio de un elaborado sistema de mecanismos de defensa y de los dispositivos tradicionales de control social. Nos desviaríamos del objetivo inmediato de este capítulo, si ofreciéramos sugerencias constructivas para llevar a cabo tales cambios en la gestión económica y doméstica, lo cual sería necesario para vencer este factor condicionante y de esta manera ofrecer oportunidades vocacionales libres a ambos sexos por igual. No será una tarea simple, sino más bien compleja, poder realizar estos cambios de manera tal que se logre la máxima mejora social. Exigirá las mejores intenciones y el esfuerzo más progresista que podamos realizar, y sólo se logrará con el correr de los años y las décadas. 
En este momento, es esencial saber si las bases para las acciones futuras se hallan ya en los hechos irrefutables de la naturaleza humana. En el estado actual del conocimiento científico sería tan dogmático ( $y$ por lo tanto igualmente indeseable) afirmar que no existen diferencias significativas entre los sexos con respecto al intelecto, como afirmar que tales diferencias sí existen. Lo único que podemos decir es que, hasta el momento, la psicología experimental no ha descubierto ninguna diferencia entre los sexos respecto a los rasgos mentales que pudiera suponer una división del trabajo basada en motivos psicológicos. El beneficio social sería enorme si el público pudiera reconocer con inteligencia que, por ahora, muchas de las preguntas acerca de las aptitudes vocacionales de las mujeres no se pueden contestar de manera definitiva debido a que nunca se ha reunido la información necesaria para formular estas respuestas. Por lo que hasta este momento se sabe, las mujeres son intelectualmente tan competentes como los hombres para seguir todas y cada una de las vocaciones humanas. 